\title{
Self-Duality and $C^{*}$ - Reflexivity of Hilbert $C^{*}$-Moduli
}

\section{Franik}

Gegenstand dieses Artikels sind eine neue Definition des Begriffes ,,selbstdualer Hilbert- $C^{*}$ Modul " als ein Kategoriebegriff der Banach-C*-Moduln und die Bedingungen für einige Hilbert- $C^{*}$-Moduln, selbstdual oder $C^{*}$-reflexiv zu sein. Es wird die Isomorphie zweier beliebiger Hilbertstrukturen auf einem gegebenen selbstdualen Hilbert- $C^{*}$.Modul gezeigt, die äquivá lente Normen zur gegebenen Norm induzieren. Fin topologisches Kriterium der Selbstdualität und $C^{*}$ :Reflexivität von Hilbert- $W^{*}$-Moduln wird bewiesen. Weiterhin wird ein Kriterium der -Selbstdualität des abzählbar erzẹgten Hilbert-C*. Mơduls $l_{2}(\mathfrak{A})$ für bęliebige $C^{*}$-Algebren $\mathfrak{A}$ gezeigt. Als eine Anwendung wird die Klassifikation der abzählbar erzeugten selbstdualén Hilbert- $W^{*}$ Moduln durch deren Struktur gegeben.

- IІредметом стати нвляется новое определеніге понятия „автодуальның гильбертов $C^{*}$. модууль" как понятие категории банаховых $C^{*}$-модулей и условия діля некоторых гијьбертовых $C^{*}$-модулей быть автолуальными иіи $C^{*}$-рефлексивными. Показана изоморф ность любых двух гильбертовых структур иа заданном автопуальном. гильбертовом

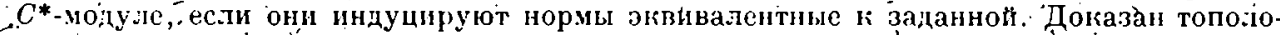
гический критерий антокуаньости и $C^{*}$-рефлексивности гильбертовых $W^{*}$-модулей.

" - Далее, 'сформулирован критерий автодуальности счетно порожценного гильбертового .-2A-модуля $l_{2}(\mathscr{A})$ для любых $C^{*}$-алгебр $\mathscr{A}$. В качестве приложения дана классификация счетно порожіценных гильбертовых $W^{*}$-модулей их структурой.

The subject of this paper are a new definition of the notion "self-dual Hilbert $C$ *-module" as a categorical" concept of Banach $C^{*}$-moduli, and the "conditions for some Hilbert $C^{*}$-moduli to be self-dual or $C^{*}$.reflexive. The isomorphism of any two Hilbert structures on a given selfdual Hilbert $C^{*}$-module inducing equivalent norms to the given' one is stated. $A$ topological - criterion of self-duality and $C^{*}$-reflexivity of Hilbert $W^{*}$-moduli is proved. A criterion of selfduality of the countably generated Hilbert $\mathfrak{T}$-module $l_{2}(\mathfrak{A})$, is stated for arbitrary $C^{*}$-algebras 2. As an application the classification of countably generated Hilbert $W^{*}$-moduli by their structure is given.

\section{$\$ 1$ Introduction}

At the beginning we fix some denotations and give certain' facts and examples from the literature: All moduli in this paper are left moduli by definition. A pre-Hilbert $\mathfrak{A}$-module over a certain $C^{*}$-algebra $\mathfrak{A}$ is an $\mathfrak{A}$-module $\mathbf{M}$ equipped with a conjugate bilinear mapping $\langle\cdot, \cdot\rangle: \mathbf{M} \times \mathbf{M} \rightarrow \mathfrak{A}$ satisfying

(i) $\langle\mathbf{x}, \mathbf{x}\rangle \geqq 0$ for any $\mathbf{x} \in \mathbf{M}$,

(ii) $\langle\mathbf{x}, \mathbf{x}\rangle=0$ if and only if $\mathbf{x} \doteq \mathbf{0}$,

(iii) $\langle\mathbf{x}, \mathbf{y}\rangle=\langle\boldsymbol{y}, \mathbf{x}\rangle^{*}$ for any $\mathbf{x}, \mathbf{y} \in \mathbf{N}$,

(iv) $\langle a \mathbf{x}, \mathbf{y}\rangle=u\langle\mathbf{x}, \mathbf{y}\rangle$ for any $u \in \mathfrak{A}, \mathbf{x}, \mathbf{y} \in \mathbf{M}$. 
The map $\langle\cdot, \cdot\rangle$ is called the $\mathfrak{A}$-valued inner product on M. Let us remark that we will write "pre-Hilbert $C^{*}$-module" instead of "pre-Hilbert $\mathfrak{A}$-module over the $C^{*}$-al-: gebra $\mathfrak{U}$ " whenerer the concrete properties of the underlying $C^{*}$-algebra $\mathfrak{A}$ are unimportant in the context. A pre-Hilbert $C^{*}$-module is $H i l b e r t$ if it is complete with respect to the norm $\|\cdot\|_{1}=\|\langle\cdot, \cdot\rangle\|_{\mathfrak{1}}^{\mathbf{2}}$. 'Two Hilbert $\mathfrak{\mathfrak { A }}$-moduli $\left\{\mathbf{I},\langle\cdot, \cdot\rangle_{\mathbf{M}}\right\},\left\{\mathbf{N},\langle\cdot, \cdot\rangle_{\mathbf{N}}\right\} \cdot$ over a certain fixed $C^{*}$-algebra $\mathfrak{A}$ are isomorphic if there exists a bijective $\mathfrak{A}$-linear bounded $\operatorname{map} B: \mathbf{I}^{-} \rightarrow \mathbf{N}$ such that $\langle\mathbf{x}, \mathbf{y}\rangle_{\mathbf{M}}=^{\prime}\langle B(\mathbf{x}), B(\mathbf{y})\rangle_{\mathbf{N}}$ for any $\mathbf{x}, \mathbf{y}^{\prime} \in \mathbf{M}$. A Hilbert $\mathfrak{A}-$ module over a certain $C^{*}$-algebra $\mathfrak{A}$ is called finitely (resp., countably, countably infinitely) generated if it is finitely (resp., countably, countably. infinitely) generated as an $\mathfrak{A}$-module, cf. [9]. A $C^{*}$-submodule $\mathbf{I}$ of a certain Hilbert $C^{*}$-module $\{\mathbf{N},\langle\cdot ; \cdot\rangle\}$, is a Hilbert $C^{*}$-submodule of $\mathbf{N}$ if $\{\mathbf{M},\langle\cdot, \cdot\rangle\}$. is a Hilbert $C^{*}$-module. A pre-Hilbert $C^{*}$ submodule $\{\mathbf{M},\langle:, \cdot\rangle\}$ of a certain pre-Hilbert $C^{*}$-module $\{\mathbf{N},\langle\cdot, \cdot\rangle\}$ is a direct summand of $\mathbf{N}$ if any element of $\mathbf{N}$ has a (unique) decomposition into the sum of an element of $\mathbf{M}$ and an element of the orthogonal with respect to $\langle\cdot, \cdot\rangle$ complement of $\mathbf{M}$.

Let us consider some examples.

(i) Any $C^{*}$-algebra $\mathfrak{A}$ becomes a Hilbert $\mathfrak{A}$-module with the inner product $\langle\cdot, \cdot\rangle_{\mathfrak{A}}$ being de. fined by $\langle a, b\rangle_{\mathfrak{T}}=a b^{*}$. for any $a, b \in \mathfrak{A}$ :

(ii) Any Hilbert space is a Hilbert $C^{*}$-module over the $C^{*}$-algebrai $\mathbb{C}$.

(iii) Let $I$ be an index set and let $\left\{\mathfrak{D}_{\alpha}\right\}_{\alpha \in l} l$ be a collection of left ideals of a certain $O^{*}$-algebra $\mathfrak{A}$ indexed by 1 . Then the set of all $I$-tuples $\mathbf{x}=\left\{x_{\alpha}\right\}_{\dot{\alpha} \in I}$ for which the sum $\sum x_{\alpha} x_{\alpha}^{*}$ converges with respect to the $\mathfrak{A}$-norm is a pre-Hilbert $\mathfrak{A}$-module.

(iv) Let $H$ be a Hilbert spice and $\mathfrak{A}^{2}$ be a $C^{*}$ algebra: The algebraic tensor product $\mathfrak{A} \otimes I$ be. comes a pre-Hilbert $\mathfrak{Y}$-module with the inner product $\langle\cdot, \cdot\rangle$, defined on elementary tensors by $\langle a \otimes \xi, b \otimes \eta\rangle=a b^{*}\langle\xi, \eta\rangle_{I I}$. Obviously, if the Hilbert space $H$ is finite-dimensional, then $\mathfrak{U} \otimes \mathbb{C}^{n}$ is isomorphic to the set $\mathfrak{A}^{n}$ of $n$-tuples of elements of $\mathfrak{A}$. Let us remark that the norm. closure of $\mathfrak{A} \otimes l_{2}$ is denoted by $l_{2}(\mathfrak{A})$, and it plays an important role describing properties of arbitrary countably generated Hilbert $\mathfrak{A}$-moduli, cf. [9: Th. 2].

(v) Let $\xi=\left(E^{\prime}, p ; K, H\right)$ be a locally trivial Hilbert bundle over a compact space $K$. Denote by $I^{\top}(\xi)$ the set of all continuous sections of this bundle. Then $\Gamma(\xi)$ becomes in a natural way a Hilbert $C(K)$-module splitting the inner products of the fibres $H_{x}, x \in K$, ef. [2: p. 48-49]:

We denote by $\mathbf{M}^{\prime}$ the set of all bounded module maps $f: \mathbf{M} \rightarrow$ A. Following $\mathbf{W}$. $\mathbf{L}$. Paschk E [14] a Hilbert $C^{*}$-module $\mathbf{M}$ is called self-dual if every map $r \in \mathbf{M}^{\prime}$ is of the form $\left\langle\cdot ; a_{r}\right\rangle$ for some $a_{r} \in$ M. W. L. Paschke proved that in the case of $\mathscr{A}$ being-a $W^{*}$. algebra the $\mathfrak{A}-$ valued inner product on a pre-Hilbert $\mathfrak{A}$-module $\{\mathbf{I},\langle\cdot, \cdot\rangle\}$ lifts to an $\mathfrak{N}$-valued inner product $\langle\cdot, \cdot\rangle_{D}$ on the Banach $\mathfrak{A}$-module $\mathbf{M}^{\prime}$ turning $\left\{\mathbf{H}^{\prime},\langle\cdot, \cdot\rangle_{D}\right\}$ into: a self-dual Hilbert $\mathfrak{A}$-module, ef. [14: Th. 3.2]. A. S. Mrščenko showed that every finitely generated Hilbert $C^{*}$-module is self-duall, ef. [12]. P. P. Saworornow got the result that every Hilbert $\mathfrak{A}$-module over a finite-dimensional $C^{*}$-algebra $\mathfrak{A}$ is selfdual; ef. [19: Th. 3].

Denote by. $\mathbf{M}^{\prime \prime}$-the $\mathfrak{A}$-module of all bounded module maps from $\dot{M}^{\prime}$ into $\mathfrak{A}$. Let $q$ be the module map $q: \mathbf{M} \rightarrow \mathbf{I}^{\prime \prime}$ defined by $q(\mathbf{m})-[r]=(r(\mathbf{m}))^{*}$ for each $\mathbf{m} \in \mathbf{M}$, any $r \in \mathbf{M}^{\prime}$. A Banach $C^{*}$-module $\mathbf{M}$ over $\mathfrak{A}$ is called $C^{*}$-reflexive (or $\mathfrak{A}$-reflexive) if the module máp $q$ is a module isomorphism, cf. [13]. For a Hilbert $C^{*}$-module the map $q$ is automatically an isometry [13: Cor. 1.1]. It turns out that for Hilbert $W^{*}$-moduli the $C^{*}$-reflexivity is equivalent to the self-duality [14: Th. 3.2]. W. L. P.Ascirke [16] proved that for any Hilbert $\mathfrak{A}$-module $\mathbb{M}$ over a certain $C^{*}$-algebra $\mathfrak{A}$ the $\mathfrak{A}$-valued inner product can be extended to the $\mathfrak{X}$-bidual Banach $\mathfrak{A}$-module $\mathbf{M}^{\prime \prime}$ turning it into a. $C^{*}$-reflexive Hilbert $\mathfrak{A}=$ module.

The paper is organized as follows: The sccond part is concerned with the definition of the notion "self-dual Hilbert $C^{*}$-module". We show that the property of a Hilbert $C^{*}$-module to be self-dual (in the sense of [14]) depends not on the structure of the given inner product, but only on the existence of an inner product on the under: 
lying Banach $C^{*}$-module inducing an equivalent Hilbert norm and realizing the condition of self-duality (Proposition 2.2). A new definition of this notion is given (Definition 2.1).describing it on the category of Banach $C^{*}$-moduli. As a consequence we get that on self-dual Hilbert. $C^{*}$-moduli any two Hilbert structures inducing equivalent norms to the given one are isomorphic (Theorem-2.6). We prove that any selfdual Hilbert $C^{*}$-submodule of an arbitrary pre-Hilbert $C^{*}$-module is a direct sum-mand (Theorem 2.7).

In the third part Hilbert $W^{*}$-moduli are treated. We characterize $C^{*}$-reflexive (and hence, self-dual) Hilbert $W^{*}$-moduli by their ininer topological properties (Theorem 3.2). Also an example of a non- $C^{*}$-reflexive Hilbert $\mathfrak{A}$-module,over a certain commutative unital $W^{*}$-algebra $\mathfrak{A}$ is given (Example 3.6) contradicting [13: Th. 2.1].

The fourth part of this paper is concerned with the Hilbert $\mathfrak{A}$-module $l_{2}(\mathfrak{A})$ over certain $C^{*}$-algebras $\mathfrak{A}, l_{2}(\mathfrak{A})$ being standard for all countably generated Hilbert $\mathfrak{X}$ moduli in the sense of [9: Th. 2]. We give a criterion of self-duality of $l_{2}(\mathfrak{A})$.(Theorem 4.3). Moreover, we show that every Hilbert $\mathfrak{A}$-module over a certain $C^{*}$-algebra $\mathfrak{A}$ is self-dual if and only if $\mathfrak{A}$ is finite-dimensional:(Proposition 4.4). As an application we get the classification of all countably generated self-dual Hilbert $W^{*}$-moduli by their structure (Proposition 4.7).

\section{$\$ 2$ The notion "self-dual Hilbert $C^{*}$-module" - a category concept}

W. L. PAŚHKE [14] and other authors $[12,18]$ have defined that a Hilbert $\mathscr{A}$-module $\{\mathbf{M},\langle\cdot, \cdot\rangle\}$ over a certain $C_{*}^{*}$-algebra $\mathcal{Q}$ is self-dual if and only if every bounded module map $f \in \mathbf{I}^{\prime}$ is of the form $\left\langle\cdot, \mathbf{a}_{f}\right\rangle$ for some $\mathbf{a}_{f} \in \mathbf{M}$. We give another definition.

Definition 2.1: A Banach $\mathfrak{U}$-module $\mathbf{M}$ over a certain $C^{*}$-algebra $\mathfrak{A}$ is called $\mathbf{a}^{\prime}$ self-dual Hilbert $\mathfrak{A}$-module over $\mathfrak{A}$ if there exists ạn $\mathfrak{A}$-ralued inner product $\langle\cdot, \cdot\rangle$ on * M with the properties:

(i) The norm induced on $\mathbf{M}$ by the $\mathfrak{A}$-valued inner product, $\langle\cdot, \cdot\rangle$ is equivalent to the 'given norm on $\mathbf{M}$.

(ii) The map $\varphi: \mathbf{M I} \rightarrow \mathbf{M}^{\prime}$ defined by the formula $\varphi(\mathbf{a})=\langle\cdot, \mathbf{a}\rangle(\mathbf{a} \in \mathbf{M})$ is surjective:

This definition seems to be weaker than the other one. In the following, however, wé prove the equivalence of both definitions. As a result we can show the categorical sense of the notion for the category of Banach $C^{*}$-moduli.

Proposition 2.2: Let: $\mathfrak{U}$ be a $C^{*}$-algebra. Let $\mathbf{M}$ be an $\mathfrak{A}$-module turning into a selfdual (in the sense of [14]) Hilbert $\mathfrak{A}$-module with the $\mathfrak{P}$-valued inner product $\langle\cdot, \cdot\rangle_{1}$, and turning into a Hilbert $\mathfrak{A}$-module with the $\mathfrak{A}$-valued inner product $\langle\cdot, \cdot\rangle_{2}$. We suppose the 'equivalence of the norms $\|\cdot\|_{1}$ and $\|\cdot\|_{2}$ on $\mathbf{M}$ and the completeness of $\mathbf{M}$ with respect to them.

'Then $\left\{\mathbf{M},\langle\cdot, \cdot\rangle_{2}\right\}$ is a self-dual (in the sense of [14]) Hilbert $\mathfrak{A}$-module and there exists a bounded $\mathfrak{A}$-linear operator $B: \mathbf{M} \rightarrow \mathbf{M}$ with the following properties:

(i). $\langle\mathbf{b}, \mathbf{a}\rangle_{2}=\langle\mathbf{b}, B(\mathbf{a})\rangle_{1}$ for any $\mathbf{a}, \mathbf{b} \in \mathbf{M}$.

(ii) $B$ is one-lo-one and, in addition, self-adjoint and positive on both $\left\{\mathrm{M},\langle\cdot, \cdot\rangle_{1}\right\}$ and $\left\{\mathbf{M},\langle\cdot, \cdot\rangle_{2}\right\}$.

(iii) $B$ has an inverse $B^{-1}$, which is bounded and $\left\{\right.$-linear. For $B^{-1}$ the properties of ilem (ii) are valid and $\langle\mathbf{b}, \mathbf{a}\rangle_{1}=\left\langle\mathbf{b}, B^{-1}(\mathbf{a})\right\rangle_{2}$ for any $\mathbf{a}, \mathbf{b} \in \mathbf{M}$.

Proof: (i) Since $\left\{\mathbf{M},\langle\cdot, \cdot\rangle_{1}\right\}$ is self-dual, for each a $\in \mathbf{M}$ there exists an element

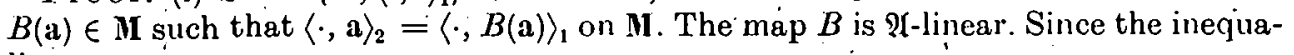
lity

$$
\|\mathbf{a}\|_{1} \leqq k\|\mathbf{a}\|_{2,} \leqq l\|\mathbf{a}\|_{1} \cdot(k, l \in(0,+\infty))^{j}
$$


is valid for any a $\in \mathbf{M}$ by supposition, and because of the inequality

$$
\begin{aligned}
\|B(\mathbf{a})\|_{1}{ }^{2} & =\|(\mathbf{B}), B(\mathbf{a})\rangle_{1}\left\|_{\mathfrak{a}}=\right\|\langle B(\mathbf{a}) ; \mathbf{a}\rangle_{2} \|_{\mathfrak{a}}^{\prime} \\
& \leqq\|B(\mathbf{a})\|_{2}\|\mathbf{a}\|_{2} \leqq l^{2}\|B(\mathbf{a})\|_{1}\|\mathbf{a}\|_{1}
\end{aligned}
$$

(cf. [14: Prop. 2.3] and (1)) we.get the boundedness of $B$. It does' not depend on the inner product.

(ii) We state the equalities $(\mathbf{a}, \mathbf{b} \in \mathbf{M})$

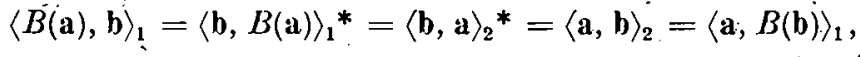

$$
\begin{aligned}
& \langle B(\mathbf{a}), \mathbf{b}\rangle_{2}=\langle B(\mathbf{a}), B(\mathbf{b})\rangle_{1}=\left\langle\mathbf{a}, B^{2}(\mathbf{b})\right\rangle_{1}=\langle\mathbf{a}, B(\mathbf{b})\rangle_{2} .
\end{aligned}
$$

This is enough to show $B=B^{*}$ with respect to both inner products. The other properties of $B$ are trivial deductions now. In particular, we get that $B$ is a one-to-one mapping.

(iii) Because of the inequality

$$
\|\mathbf{a}\|_{1}{ }^{2} \leqq k^{2}\|\mathbf{a}\|_{2^{2}}{ }^{\cdot}=k^{2}\left\|\langle\mathbf{a}, \mathbf{a}\rangle_{2}\right\|_{26}=k^{2} \cdot\left\|\langle\mathbf{a}, B(\mathbf{a})\rangle_{1}\right\|_{\mathfrak{M}} \leqq k^{2}\|B(\mathbf{a})\|_{1}\|\mathbf{a}\|_{1}
$$

being valid for any a $\in \mathbf{M}$ (cf. (1) and [14: Prop. 2.3]), we get the connection

$$
\|\mathbf{a}\|_{1} \leqq k^{2}\|B(\mathbf{a})\|_{1} \leqq k^{2}\|B\|_{0 p, 1}\|\mathbf{a}\|_{1} \text {. for any } \mathbf{a} \in \mathbf{M} \text {. }
$$

Since $B$ is bounded this means that every norm-fundamental sequence of the range of $B$ has a (unique) norm-fundamental sequence of $\mathbf{M}$ as. its pre-image. Moreover, $B$ maps the limit of this pre-image sequence into the limit of the sequence taken in the range of $B$. Consequently, since $B$ is $\mathfrak{A}$-linear the range $\operatorname{Im}(B)$ is a norm-closed $\mathfrak{A}$-submodule of $\mathbf{M}$ independent of the inner product. The Banach $\mathfrak{A}$-module Im (B) becomes a Hilbert $\mathfrak{N}$-submodule of $\mathbf{I I}$ with both inner products $\langle\cdot, \cdot\rangle_{1}$ and $\langle\cdot, \cdot\rangle_{2}$. Now there are three possibilities; how $\operatorname{Im}(B)$ can be related to the Hilbert $\mathfrak{A}$-module $\mathbf{M}$ :

(a) $\operatorname{Im}(B) \neq \mathbf{I}, \quad \operatorname{Im}(B) \perp \neq\{0\}$.

(b) $\operatorname{Im}(B) \neq \mathbf{I}, \quad \operatorname{Im}(B)^{\perp} \equiv\{0\}$

(c) $\operatorname{Im}(B) \equiv \mathbf{M}$.

We will show that, in fact, only (c) can be: To rule out the first possibility we take an element $\mathbf{b} \in \operatorname{Im}(B)^{\perp}$ with respect to $\langle\cdot, \cdot\rangle_{1}, \mathbf{b} \neq \mathbf{0}$. Then we get $\langle\mathbf{b}, \mathbf{b}\rangle_{2}=\langle\mathbf{b}, \boldsymbol{B}(\mathbf{b})\rangle_{1}$ $=0$ and, therefore, $\mathbf{b}=\mathbf{0}$. 'This is a contradiction. The' samè happens if we take $\mathbf{b} \in \operatorname{Im}(B)^{\perp}$ with respect to $\langle\cdot, \cdots\rangle_{2}, \mathbf{b} \neq \mathbf{0}$. We get $0=\langle B(\mathbf{b}), \mathbf{b}\rangle_{2}=\langle B(\mathbf{b}), B(\mathbf{b})\rangle_{1}$ and, therefore, $B(\mathbf{b})=0$. Since $B$ is injective, $\mathbf{b}^{\circ}=\mathbf{0}$ in contradiction to our choice of $\mathbf{b}$.

To drop the second possibility we use the fact that the canonical embedding of a Hilbert $\mathfrak{A}$-module $\{\mathbf{N} ;\langle\cdot, \cdot\rangle\}$ over a certain $C^{*}$-algebra' $\mathfrak{A}$,into its $\mathfrak{A}$-bidual Banach $\mathfrak{A}$-module $\boldsymbol{N}^{\prime \prime}$ does not deperid on the structure of the $\mathfrak{A}$-valued inner product. $\langle\cdot, \cdot\rangle$ by definition. Using on $\mathbf{M}$ the inner product $\left\langle\dot{ }^{\prime}, \cdot\right\rangle_{\mathbf{1}}$ wé get $\mathbf{M} " \equiv \mathbf{M}$ and $\operatorname{Im}(B)^{\prime \prime} \equiv \mathbf{M}$ as Banach $\mathfrak{A}$-moduli. Now we define on $\operatorname{Im}(B)$ a third $\mathfrak{A}$-valued inner product by the formula $\langle\mathbf{a}, \mathbf{b}\rangle_{3}=\left\langle B^{-1}(\mathbf{a}), B^{-1}(\mathbf{b})\right\rangle_{1}$ for any $\mathbf{a}, \mathbf{b}^{\prime} \in \operatorname{Im}(B)$. It is well defined since $B$ is a one-to-one, surjective, $\mathfrak{A}$-linear, bounded mapping from M onto $\mathrm{Im}(B)$. Because of (3) we get that $\left\{\operatorname{Im}(B),\langle\cdot, \cdot\rangle_{3}\right\}$ is a Hilbert $\mathfrak{A}$-module, which is, moreover, self-dual-in the sense of [14]. Using the inner product $\langle\cdot,\rangle_{3}$ we consider $\operatorname{Im}(B)^{\prime \prime} \equiv \operatorname{Im}(B)$. This means $\operatorname{Im}(B) \equiv \mathbf{M}$ in contradiction to $(\mathrm{b})$.

Therefore, only the relation $\mathbf{M} \equiv \operatorname{Im}(B)$ is possible. Moreover, the bounded $\mathfrak{A}$-lineár.operator $B^{-1}: \mathbf{M} \rightarrow \mathbf{M}$, which is inverse to $B$, exists and satisfies the conditions of the items (ii) and (iii). Finally, we show the self-duality of $\left\{\mathbf{M} ;\langle\cdot, \cdot\rangle_{2}\right\}$-in the 
sense of [14]. We choose an $r \in \mathbf{M}^{\prime}$ arbitrarily. By supposition there exists an element $\mathbf{b}_{r} \in \mathbf{M}$ such that $r(\cdot) \equiv\left\langle\cdot, \mathbf{b}_{r}\right\rangle_{\mathbf{1}}$. on $\mathbf{M}$. We define $\mathbf{c}_{r}=B^{-1}\left(\mathbf{b}_{r}\right) \in \mathbf{M}$ and get $r(\cdot) \equiv\langle\cdot, \mathbf{c}\rangle_{2}$ on $\mathbf{I I}$. Since $r \in \mathbf{M}^{\prime}$ is choosen arbitrarily, we are done

'Corollary' 2.3: Lèt $\{\mathbf{M} ;\langle\cdot, \cdot\rangle\}$ be à self-dual Hilbert $\mathfrak{A}$-module ovér a $C^{*}$-algebra $\mathfrak{A}$. Then for any $\mathfrak{A}$-valued inner product $\langle\leftarrow, \cdot\rangle_{0}$ on $\mathbf{M}$ inducing an equivalent norm to the given one there exists one and only one bounded $\mathfrak{A}$-linear invertible positive opérator $B_{0}$ on $\{\mathbf{I},\langle\cdot, \cdot\rangle\}$ with the property $\langle\cdot, \cdot\rangle_{0} \equiv\left\langle\cdot, B_{0}(\cdot)\right\rangle$, on $\mathbf{M}$. And vice versa.

This follows from [10: Temma 2, Th. 3] and from Proposition 2.2.

Corollary 2.4: Under the suppositions formulated in Proposition 2.2 beside the ine-' quality (1) there holds the inequality.

$$
\langle\mathbf{a}, \mathbf{a}\rangle_{1} \leqq C\langle\mathbf{a}, \mathbf{a}\rangle_{2} \leqq D\langle\mathbf{a}, \mathbf{a}\rangle_{1}
$$

for some constants $C, D \in(0,+\infty)$ and for uny a $\in$ iI.

Proof: We have, $\langle\mathbf{a}, \mathbf{a}\rangle_{2}=\langle\mathbf{a}, B(\mathbf{a})\rangle_{\mathbf{i}}=\left\langle B^{1 / 2}(\mathbf{a}), B^{1 / 2}(\mathbf{a})\right\rangle_{1} \leqq\left\|B^{1 / 2}\right\|_{0 p .1}^{2}\langle\mathbf{a}, \mathbf{a}\rangle_{1}$ $\doteq\|B\|_{0,1}\langle\mathbf{a}, \mathbf{a}\rangle_{1}$ for any $\mathbf{a} \in$ M (cf. [14: Prop. 2.8]). In the same way we get $\langle a, a\rangle_{1}$ $\leqq\left\|B^{-1}\right\|_{0 j, 2}\langle\mathbf{a}, \mathbf{a}\rangle_{2}$ for any a $\epsilon \mathbf{H}$

Corollary 2.5: The operalor described in l'roposition 2.2 has the property $k^{-2}$ $\leqq\|B\|_{0 p ; 1} \leqq l^{2}$, where $k, l \in \mathbb{R}$ are taiken from (1).

This follows from (2) and (3). The following corollary is suggested from [13: Th. 1.1, Cor. 1.2], where a special case of it is stated.

Theorem 2:6; Lei' $\mathfrak{A}$ be a $C^{*}$-algebra and $\left\{\mathbf{M},\langle\cdot, \cdot\rangle_{1}\right\}$ be a self-dual Hilbert $\mathfrak{A}$-module. Then every $\mathfrak{A}$-valued inner product $\langle\cdot, \cdot\rangle_{2}$ on $\mathrm{II}$, the norm induced from which is equivalent to the given norm, defines a.Hilbert structure on $\mathbf{1 1}$ isomorphic to the Hilbert structure given by. the $\mathfrak{A}$-valued inner product $\langle\cdot, \cdot\rangle_{1}$.

Proof: By Proposition 2.2 there exists a bounded $\mathfrak{A}$-linear invertible self-adjoint positive operator $B: \mathbf{l} \rightarrow \mathbf{M}$ satisfying the equality $\langle\mathbf{a} ; \mathbf{b}\rangle_{2}=\langle\mathbf{a}, B(\mathbf{b})\rangle_{\mathbf{1}}$ for any $\mathbf{a}, ' \mathbf{b} \in \mathbf{M}$., The set of all bounded $\mathfrak{A}$-linear operators on a self-dual Hilbert $\mathfrak{A}$-module is a $C^{*}$-algebra by [14: Cor. 3.5], and an operator $B$ is positive on the Hilbert $\mathfrak{A}$-module-if and only if it is positive as an element of this $C^{*}$-algebra by [10: Lemma 2, Th. 3]. So we can find a bounded $\mathfrak{A}$-linear invertible self-adjoint positive operator $C: \mathbf{M} \rightarrow \mathbf{M}$ satisfying the equality $\langle\mathbf{a}, \mathbf{b}\rangle_{2}=\langle C(\mathbf{a}), C(\mathbf{b})\rangle_{2}$ for any $\mathbf{a}, \mathbf{b} \in \mathbf{M}$

Remark 2.7: If the underlying $C^{*}$-algebra $\mathfrak{A}$ is commitative, then any Hilbert $\mathfrak{A}$-module has a unique (up to isomorphism) Hilbert structure, i.e. the propert $y^{h}$ to he self-dual is omittable in this case. This fact can be drawn from the investigations of M. J'. Dupré and R. M. Gillette [3: pp. 48-49]. However, if we drop the commutativity condition on $\mathfrak{A}$, the analogous problem of uniqueness is still opèn. There seems to be some hope to solve it affirmatively.

- Now we are able to obtain a very important property of self-dual Hilbert $C^{*}$-moduli: Tet ís previously remark that not any Hilbert $C^{*}$-submodule of a pre-Hilbert $C^{*}$. - module has to be a direct summand, in general.

Theorem 2.8: Let $\mathfrak{2}$ be a $C^{*}$-algebra, $\{\mathbf{N},\langle\cdot, \cdot\rangle\}$ be any pre-Hilbert $\mathfrak{H}$-module and . $\mathbf{I I} \subseteq \mathbf{N}$ be a self-dual Hilbert $\mathfrak{2}$-submodule. Then $\mathbf{N} \equiv \mathbf{I} \oplus \mathbf{M}{ }^{\perp}$.

Proof:We take the $\mathfrak{U}$-valued inner product on $\mathbf{M}$ given by that one on $\mathbf{N}$ reduced to $\mathbf{M} \subseteq \mathbf{N}$. By ${ }^{`}$ 14: Prop. 3.4] the injective isometric $\mathfrak{P}$-linear embedding $T: \mathbf{M} \rightarrow \mathbf{N}$ has 
an adjoiṇt $\mathfrak{A}$-linear bounded operator $T^{*}: \mathbf{N} \rightarrow \mathbf{M}$ defined on $\mathbf{N}$ such that

$$
\langle T(\mathbf{m}), \mathbf{n}\rangle_{\mathbf{N}}=\left\langle\mathbf{m}, T^{*}(\mathbf{n})\right\rangle_{\mathbf{M}} .
$$

for any $m \in \in$ II, any $n \in N$. Because of the choice of the Hilbert structure on $\mathbf{M}$ and since $T$ is isometric we cau rewrite (4) as $\left\langle T(\mathbf{m}), \mathbf{n}-T_{i}\left(T^{*}(\mathbf{n})\right)\right\rangle_{\mathbf{N}} \stackrel{=}{=} O$ for any $n^{\prime} \in \mathbf{N}$, any. $m \in \mathbf{M}$. That is, any element $n \in \mathbf{N}$ can be decomposed $n=T T^{*}(\mathbf{n})$ ${ }^{-}\left(\mathbf{n}-T^{\prime} T^{*}(\mathbf{n})\right)$, where $T^{\prime} T^{*}(\mathbf{n}) \epsilon, \mathbf{M} \subseteq \mathbf{N}$ and $\left(\mathbf{n}-T^{*}(\mathbf{n})\right) \in \mathbf{M}^{1}$. This decompo-' sition is unique

Finishing this paragraph we list some results from the literature to illustrate the importance of Theorem 2.8 .

Corollary 2.9 [13: Cor. 1.4], [2: Prop. 1], [8]: The following is true:

(i) Lei $\mathfrak{A}$ be any $C^{*}$-algebra. Let $\{\mathbf{M},\langle\cdot, \cdot\rangle\}$ be a finitely generated Hilbert $\mathfrak{A}$-submodule of an arbitrary,pre-Hilbert $\mathfrak{A}$-module $\{\mathbf{N},\langle\cdot, \cdot\rangle\}$. Then $\mathbf{N}=\mathbf{M} \oplus \mathbf{M}{ }^{1}$.

(ii) Let́ $\mathfrak{A}$ be a finité-dimensional $C^{*}$-algebra: Let $\{\mathbf{M},\langle\cdot, \cdot\rangle\}$ be a $\dot{H}$ ilbert $\mathscr{A}$-submodule . of an arbitrary pre-Hilbert $\mathfrak{A}$-module $\{\mathbf{N},\langle\cdot, \cdot\rangle\}$. Then $\mathbf{N}=\mathbf{M} \oplus \mathbf{M}{ }^{\perp}$.

\section{$\$ 3$ A topological characterization of self-dual and $C^{*}$-reflexive Hilbert $W^{*}$-moduli}

The aim of the $p$ present paragraph is to characterize self-duality and $C^{*}$-reflexivity for a special class of $I$ ilbert $C^{*}$-moduli, namely, for Hilbert $W^{*}$-moduli, by their inner topological properties. The possibility of such a characterization is based either on Theorem 2.6 for self-duality or on the definition of the notion for $C^{*}$-reflexivity. The ideas for the following investigations arise from the proving technics and from the mental background of two papers of W. L. PAschke $[14, \cdot 16]$.

Definition 3.1 : Let $\mathfrak{A}$ be a $W^{*}$-algebra, $\{\mathbf{M},\langle\cdot, \cdot\rangle\}$ be a pre-Hilbert $\mathfrak{A}$-module and $P$ be the set of all normal states on $\mathfrak{A}$. The topology induced on $\mathbf{M}$ by the seminorms

$$
f(\langle\cdot, \cdot))^{1 / 2}, \quad f \in P
$$

( is denoted by $\tau_{1}$. The topology induced on $\mathbf{M}$ by the linear functionals $f(\langle\cdot, \mathbf{y}\rangle), f \in P$, $\mathbf{y} \in \mathbf{M}$, is dénoted by $\tau_{2}$.'

Let us'remark, that the topology $\tau_{2}$ was already'explicitly defined by W. L. PAschke in [14: Remark 3.9], whereas the topology $\tau_{1}$ was suggested to the author by the proving technics of [16: Iscmma 2.3]. If we define an ' $\mathfrak{A}$-valued inner product on the $W^{*}$ algebra $\mathfrak{A}$ by the formula $\langle a, b\rangle_{\mathfrak{N}}=a b^{*}, \dot{u}, b \in \mathfrak{A}$, the topology $\tau_{2}$ coincides with the weak*, topology on $\mathfrak{A}$. In the case of $\mathfrak{A}$ being $\mathbb{C}$ and $\mathbb{I}$ being an arbitrary Hilbert space the topology $\tau_{1}$ is the Hilbert topology on $\mathbf{M}$, but the topology $\tau_{2}$ is the weak and weak* topology on M. That is, they do not coincide, in general.

Theorem 3.2: Let $\mathfrak{A}$ be a $W^{*}$-algebra and $\{\mathbf{M},\langle\cdot, \cdot\rangle\}$ be a Hilbert $\mathfrak{A}$-module. The following conditions for $\mathbf{M}$ are equivalent:

(i) $\mathbf{M}$ is self-dual.

(ii) $\mathbf{M}$ is. $\mathfrak{A}$-reflexive.

(iii) The unit ball of $\mathbf{M}$ is $\tau_{1}$-complete.

(iv) The unit ball of $\mathbf{M}$ is $\tau_{2}$-complete.

Proof: (i) $\Leftrightarrow$ (ii) follows from the definitions and from [14: Th. 3.2]. (i) $\Rightarrow$ (iii): Assime that the unit ball of a self-dual Hilbert $\mathfrak{A}$-module $\mathbf{M}$ is not complete relative to the topology $\tau_{1}$ : Denote by $\mathbf{L}$ the linear hull of the completion of the unit ball of 
$\mathbf{M}$ relative to the topology $\tau_{1}$. For the extensions of the semi-norms (5) from $\mathbf{M}$ to $\mathbf{L}$ we use the same denotations. By assumption there exists an $r \in \mathbf{L} \backslash \mathbf{M}$ and $\dot{a}$ normboúnded net $\left\{y_{a}\right\}_{\alpha \in I} \subset M$ such that for every $f \in P$ and for each $\varepsilon>\dot{O}$ there is án $\alpha \in I$ with $f\left(\left\langle r-y_{\beta}, r-y_{\beta}\right\rangle\right)^{\circ}<\varepsilon$ for any $\beta \geqq \alpha$. We fix $f^{\prime} \in P, \varepsilon>O, \alpha \in I$ and an arbitrary $\mathbf{x} \in \mathbf{M I}$. Then

$$
\begin{aligned}
\left|f\left(\left\langle\mathbf{x}, \mathbf{y}_{\beta}\right\rangle\right)-f\left(\left\langle\mathbf{x}, \mathbf{y}_{\gamma}\right\rangle\right)\right| & =\left|f\left(\left\langle\mathbf{x}, \mathbf{y}_{\beta}-\mathbf{y}_{\gamma}\right\rangle\right)\right| \\
\cdot & \leqq f(\langle\mathbf{x}, \mathbf{x}\rangle)^{1 / 2} f\left(\left\langle\mathbf{y}_{\beta}-\mathbf{y}_{\gamma}, \mathbf{y}_{\beta}-\mathbf{y}_{\gamma}\right\rangle\right)^{1 / 2} \leqq(2 \varepsilon f(\langle\mathbf{x}, \mathbf{x}\rangle))^{i / 2}
\end{aligned}
$$

for any $\bar{\beta}, \dot{\gamma} \geqq \dot{\alpha}$. Consequently, there exists

$$
w^{*} \leq \lim \left\{\left\langle\mathbf{x}, \mathbf{y}_{\mathrm{a}}\right\rangle: \alpha \in I\right\} \stackrel{\rightleftharpoons}{\risingdotseq} \dot{R}(\dot{\mathbf{x}})
$$

for each $\dot{x} \in \mathbf{M}$. Furthermore, the inequality

$$
\left|f\left(\left\langle\mathbf{x}, \mathbf{y}_{\beta}\right\rangle\right)\right| \leqq\|\mathbf{x}\| \sup \left\{\left\|\mathbf{y}_{a}\right\|: \alpha \in I\right\},(\beta \in I) \backslash .
$$

shows'the boundedness of the map $R: \mathbf{M} \rightarrow \mathfrak{A}$ defined by (6). The $\mathscr{A}$-linearity of $R$ is obvious. Thus, (6). defines a bounded module map $R$. By assumption there exists an element $\mathbf{z} \in \mathbf{M}$ such that $R(\mathbf{x})=\langle\mathbf{x}, \mathbf{z}\rangle$-for any $\mathbf{x} \in \mathbf{M}$. Consequently, we arrive at $w^{*}-\lim \left\{\left\langle\mathbf{x}, \mathbf{y}_{\beta}\right\rangle: \beta \in I\right\}=\langle\mathbf{x}, \mathbf{z}\rangle$ for any $\mathbf{x} \in \mathbf{M}, \mathbf{z} \in \mathbf{I}$ being the $\tau_{\mathbf{1}}$-limit of the normbounded net $\left\{\mathbf{y}_{\mathbf{a}}\right\}$. This means $r=\mathbf{z} \in \mathbf{M}$ in contradiction to our assumption.

(ii) $\Rightarrow$ (i): We take an arbitrary $r \in \mathbf{M}^{\prime}$ and we suppose the $\tau_{1}$-completeness of the unit ball of M. By [14:Th. 3.2] we can lift the $\mathfrak{A}$-valuedinńer product $\langle\cdot, \cdot\rangle$ from M to $\mathbf{M}^{\prime}$ turning $\mathbf{M}^{\prime}$ into a self-dual Hilbert $\mathfrak{A}$-module and satisfying the following 'properties for the lifted inner product $\langle\cdot, \cdot\rangle_{D}$ :

$$
\begin{array}{lll}
\langle\mathbf{x}, \mathbf{y}\rangle=\langle\varphi(\mathbf{x}), \varphi(\mathbf{y})\rangle_{D} & \text { if } & \mathbf{x}, \mathbf{y} \in \mathbf{M}, \\
r(\mathbf{x})=\langle\varphi(\mathbf{x}), r\rangle_{D} & \text { if } & \mathbf{x} \in \mathbf{M}, r \in \mathbf{M}^{\prime} \backslash \mathbf{M},
\end{array}
$$

where $\phi(\mathbf{y})=\langle\cdot, \mathbf{y}\rangle$ for any $\mathbf{y} \in \mathbf{M}$. Furthermore, $\langle\mathbf{M}, \mathbf{M}\rangle$ and $\left\langle\mathbf{M}^{\prime}, \mathbf{M}^{\prime}\right\rangle_{D}$ are $W^{*}$-algebras, $\left\langle\mathbf{M I}^{\prime}, \mathbf{M}\right\rangle$ being a two-sided ${ }^{*}$-ideal in $\left\langle\mathbf{M}^{\prime}, \mathbf{M}^{\prime}\right\rangle_{D}$. Therefore, they coincide because of the properties of the lifted inner product, and $\langle r, r\rangle_{D}$ belongs to $\langle\mathbf{M}, \mathbf{M}\rangle$. First, let $\mathfrak{A}$ be $\sigma$-finite and let $g \in, P$ be a faithful normal state on $\mathfrak{A}$, which exists according to [1: p: 94, Prop. 2.3.6]. Let $\{H, \pi, \Omega\}$ be the cyclic representation associated with $g$ : The vector $\Omega \in H$ is both cyclic and separating. The linear space $\mathbf{M}$ equipped with the inner product $g(\langle\cdot, \cdot\rangle)$ turns into a pre-Hilbert space. The map $g(r(\cdot)): \mathbf{M} \rightarrow \mathbb{C}$ is a linear functional on it. Consequently, there exists an element $r_{g}$ in the completion of $\mathbf{M}$ relative to the norm $g(\langle\cdot, \cdot\rangle)^{1 / 2}$ such that $g\left(\left\langle\mathbf{x}, r_{g}\right\rangle\right)=g(r(\mathbf{x}))$ for any $\mathbf{x} \in$ : II. That means'there exists a sequence $\left\{x_{i}\right\}_{i \in \mathbb{S}} \subset \mathbf{M}$ such that

$$
\begin{aligned}
0 & =\lim _{i \rightarrow \infty} g\left(\left\langle\mathbf{x}_{i}-r_{g}, \mathbf{x}_{\mathbf{i}}-r_{g}\right\rangle\right) \\
& =\lim _{i \rightarrow \infty} g\left(\left\langle\varphi\left(\mathbf{x}_{i}\right)-r, p\left(\mathbf{x}_{i}\right)-r\right\rangle_{D}\right) \\
& =\lim _{i \rightarrow \infty}\left\|\left[\pi\left(\left\langle\varphi\left(\mathbf{x}_{i}\right)-r, \varphi\left(\mathbf{x}_{i}\right)-r\right\rangle_{D}\right)\right]^{1 / 2} \Omega\right\|^{2} .
\end{aligned}
$$

Since the vector $\dot{\Omega} \in H$ is both cyclic and separating there exists $w^{*}-\lim _{i}\left\langle\varphi\left(\mathbf{x}_{i}\right)-r\right.$, $\left.\varphi\left(x_{i}\right)-r\right\rangle_{D}=0$ by [1: Lemma 2.5.38, Lemma 2.5.39].' This proves the implication in the case of $\mathfrak{A}$ being $\sigma$-finite.

If $\mathfrak{A}$ is not $\sigma$-finite, there exists an increasing directed net of projections $\left\{p_{a}\right\}_{\alpha \in I}$ $\subset \mathfrak{U}$ such that $p_{\mathfrak{a}} \mathfrak{I} p_{\mathfrak{a}}$ is a $\sigma$-finite $W^{*}$-algebra for each $\alpha \in I$ and $w^{*}-\lim p_{\mathfrak{a}}=1_{\mathfrak{Q}}$. 
(cf. [1: p. 164]). Observing $p_{a} \mathfrak{H} p_{a}$ and $\left(p_{a} r\right) \in \mathbf{M}^{\prime}$ for each $\alpha \in I$ we 'conclude that $\left(p_{\alpha} r\right) \epsilon^{\cdot} \mathbf{M}$ for each $\alpha \in I$ since $\left\langle p_{\alpha} r, p_{\alpha} r\right\rangle_{D}=p_{a}\langle r, r\rangle_{D} p_{\alpha}^{*}$ and the latter belongs to $p_{a} \mathfrak{A} p_{a}$ for each $\alpha \in I$. Consequently, the $\tau_{1}$-limit of the bounded net $\left\{p_{a} r\right\}_{\alpha_{\epsilon} I}$ belongs to $\mathbf{M}$ and it is equal to $r \in \mathbf{M}^{\prime}$. So the self-duality of $\mathbf{M}^{\prime}$ turns out.

(iii) $\Leftrightarrow$ (iv): First, if the unit ball of $\mathbf{M}$ is $\tau_{1}$-complete, the $\mathbf{M}$ must be self-dual as shown above. By [14: Prop. 3.8, Remark 3.9] there follows that $\mathrm{MI}$ is a conjugate space. with weak* topology $\tau_{2}$. Therefore, the unit ball of $\mathbf{M}$ is $\tau_{2}$-complete. Secondly, let $\left\{\mathbf{x}_{\mathrm{a}}\right\}_{\mathrm{a}_{\in} I} \subsetneq \mathbf{M}$ be a-norm-bounded $\tau_{1}$-fundamental net and let the unit ball of $\mathbf{M}$ be $\tau_{\mathbf{2}}$-complete. Then, for any $\mathbf{y} \in \mathbf{M}, f \in P, \beta, \gamma \dot{\epsilon} I$,

$$
\left|f\left(\left\langle\mathbf{x}_{\beta}, \mathbf{y}\right\rangle\right)-f\left(\left\langle\mathbf{x}_{y}, \mathbf{y}\right\rangle\right)\right|^{2} \leqq f\left(\left\langle\mathbf{x}_{\beta}-\mathbf{x}_{y}, \mathbf{x}_{\beta}-\mathbf{x}_{\gamma}\right\rangle\right) f(\langle\mathbf{y}, \mathbf{y}\rangle)
$$

Denote' by $\mathbf{L}$ the linear hill of the $\tau_{1}$-completion of the unit ball of $\mathbf{M}$. The limit $\tau_{1}-\lim \mathbf{x}_{a} \doteq \ell$ exists in $\mathbf{L}$. From the inequality $(7)$ we get that the net $\left\{\mathbf{x}_{a}\right\}$ is also $\tau_{2}$-fundamental and so the $\tau_{2}$-limit $x \in \mathbf{M}$ exists by assumption. Recall that $\mathbf{I}=\mathbf{M}^{\prime}$. and that the $\mathfrak{A}$-valued inner product lifts from-M to $\mathbf{M}^{\prime}$ turning $\mathbf{M}^{\prime}$ into a self-dual Hilbert $\mathfrak{A}$-module. Thus, $t=\tau_{1}-\lim \mathbf{x}_{\alpha}=\tau_{2}-\lim \mathbf{x}_{\alpha} \stackrel{x}{=} \in \mathbf{M}$

Remark 3.3: Let $\mathfrak{A}$ be an infinite-dimensional $\sigma$-finite $W^{*}$-algebra and $\{\mathbf{M},\langle\cdot, \cdot\rangle\}$ be a Hilbert $\mathfrak{A}$-module. If $g$ is a faithful normal state on $\mathfrak{A}$, the Hilbert completion of the pre-Hilbert space $\{\mathbf{M}, g(\langle\cdot, \cdot\rangle)\}$ does not coincide with the Hilbert $\mathfrak{A}$-module $\mathbf{M}$, in general:

Corollary 3.4: If $\mathfrak{\mathfrak { A }}$ is a $W^{*}$-algebra und $\mathbf{M}$ is a self-dual Hilbert $\mathfrak{A}$-module, then the $C^{*}$-algebra $\langle\mathbf{M}, \mathbf{M}\rangle$ is a $W^{*}$-subalgebra of $\mathfrak{A}$ and a two-sided ideal in $\mathfrak{X}$.

The converse is not true, in general, as will be shown on the example of $l_{2}(\mathfrak{A})$ in $\S 4$ of the present paper.

Corollary $3.5:$ Let $\mathfrak{A}$ be an infinite-dimensional $C^{*}$-algebra having $a W^{*}$-subalgebra $\mathfrak{B}^{\prime}$ as its two-sided ideal. Let $\mathbf{M}$ be a finitely generated Hilbert $\mathfrak{A}$-module and $\mathbf{N}$ be an arbitrary Hilbert $\mathscr{B}$-module. Then the direct sum $\mathbf{M} \oplus \mathbf{N}^{\prime}$ becomes a self-dual Hilbert , $\mathfrak{A}$-module.

Proof: First; we note that $\boldsymbol{N}$ is a Hilbert ' $\mathfrak{A}$-module; too, since $\mathfrak{B}$ is a two-sided ideal in $\mathfrak{A}$. Furthermore, the set of bounded module maps $f: \mathbf{N} \rightarrow, \mathfrak{A}$ coincides with $\mathbf{N}^{\prime}$ because $f(\mathbf{n})=f\left(1_{\mathfrak{B}} \mathbf{n}\right)=\mathbf{1}_{\mathfrak{B} f} f(\mathbf{n}) \in \mathfrak{B}$ for any $\mathbf{n} \in \mathbf{N}$. We know from [12] that $\mathbf{M}$ is self-dial. $\mathbf{N}^{\prime}$ is also self-dual as it was shown in [14: Th. 3.2]

Example 3.6: Take $\mathfrak{A}=l_{\infty}, \dot{\mathbf{I}}=c_{0}$ with the $\mathfrak{A}$-valued inner product $\langle a, \dot{b}\rangle_{\mathfrak{T}}$ $=a b^{*}$, for $a, b \in c_{0}$. Easy computations show that $\mathbf{I}^{\prime}=l_{\infty}$ and $\mathbf{M} \mathbf{1}^{\prime \prime}=l_{\infty}$. This is an elegant counter-example to [13: Th. 2.1].

For the completeness of the present paragraph we reproduce a result of W. L. PAscrik concerning another criterion of self-duality and $C^{*}$.reflexivity of Hilbert $W^{*}$-moduli.

Definition 3.7 [14]: Let $\mathfrak{A}$ be a $W^{*}$-algebra, $I$ be an index set and $\left\{M_{\alpha},\langle\because ;\rangle_{\alpha \in I}\right.$ be a collection of pre-Hilbert $\mathscr{A}$-moduli indexed by $I$. Let $F$ denote the set of finite subsets of $\dot{I}$, directed upwards:býy inclusion. For I-tuples $\mathbf{x}=\left\{\mathbf{x}_{a}\right\}, \mathbf{y}=\left\{\mathbf{y}_{a}\right\},\left(\mathbf{x}_{\alpha}, \mathbf{y}_{\alpha} \in \mathbf{M}_{a}\right)$ and $S \in F$, we set

$$
\langle\mathbf{x}, \mathbf{y}\rangle_{S}=\sum_{\alpha \in S}\left\langle\mathbf{x}_{\alpha}, \mathbf{y}_{\mathbf{a}}\right\rangle:
$$

Let $\mathbf{M}$ denote the set of $I$-tuples $\mathbf{x}=\left\{\mathbf{x}_{a}\right\}$ such that $\sup \left\{\langle\mathbf{x}, \mathbf{x}\rangle_{S}: S \in F\right\}$ is finite. Notice that for $\mathbf{x} \in \mathbf{M}$ the net $\left\{\langle\mathbf{x}, \mathbf{x}\rangle_{S}\right\}_{S \in F}$ is bounded in norm and increasingly directed. We let $\langle\mathbf{x}, \mathbf{x}\rangle$ denote its least upper bound. 'The net $\left\langle\langle\mathbf{x}, \mathbf{y}\rangle_{S}\right\}_{\in \in F}$ is also bounded and $w^{*}$-convergent for any $\mathbf{x}, \mathbf{y} \in \mathbf{M}$. We denote by $\langle\mathbf{x}, \mathbf{y}\rangle$ its $w^{*}$.limit. Under co-ordinatewise operations $\mathbf{M}$ is a left. $\mathscr{A}$-module, and $\langle\cdot, \cdot\rangle$ defined as above is an $\mathfrak{A}$-valued inner product on $\mathbf{M}$. We call the pre-Hilbert $\mathscr{A}$-module $\left\{\mathbf{M},{ }^{\prime}\langle\cdot, \cdot\rangle\right\}^{\prime}$ the ultravea $k$ direct sum of the moduli $\left\{\mathbf{M}_{a},\langle\cdot, \cdot\rangle\right\}$ and write $\mathbf{M}=\operatorname{UDS}\left\{\mathbf{M}_{a}: \alpha \in I\right\}$. 
Theorem 3.8 [14: Th. 3:12]: Let $\mathfrak{A}$ be $a W^{*}$ algebra and $\mathbf{M}$ be a Hilbert $\mathfrak{A}$-module. Then the following two conditions for $\mathbf{M}$ are equivalent:

(i) M is self-dual.

(ii) There is a collection $\left\{p_{\alpha}\right\}_{\alpha \in I}$ of (not necessiary distinct) non-zero projections of 9 such that $\mathbf{M}$ and UDS $\left\{\mathfrak{A} p_{a}: \alpha \in I\right\}$ are isomorphic as Hilbert $\mathfrak{A}$-moduli.

Remark 3.9: This theorem suggests a possibility to construct other useful topologies on self-dual Hilbert $W^{*}$-moduli $\mathbf{M}$ in the following way: One must take a topology on the underlying $W^{*}$-algebra $\mathfrak{A}$ with respect to which the unit ball of $\mathfrak{A}$ is complete. Then one has to combine this topology either with the map $\langle\cdot, \cdot\rangle$ on $\mathbf{M} \times \mathbf{M}$ or with all $\mathfrak{A}$-linear bounded functionals of $\mathbf{M}^{\prime}$. We could get topologies on $\mathbf{M}$ with respect to which the unit ball of $\mathbf{M}$ would be complete.

\section{$\$ 4$ A criterion of self-duality of $l_{2}(\mathfrak{H})$. Applications}

Let $\mathfrak{A}$ be an arbitrary $C^{*}$-algebra. We consider the Hilbert $\mathfrak{A}$-module $l_{2}(\mathfrak{A})$ mentioned in the introduction. It is representable as the set of all sequences $\mathbf{a}=\left\{a_{i}\right\}_{i \in \mathbb{N}} \subset \mathfrak{A}$ for which the series $\sum a_{i} a_{i}{ }^{*}$ converges relative to the norm topology in $\mathfrak{U}$. The inner product on it is defined as $\langle\mathbf{a}, \mathbf{b}\rangle=\sum a_{i} b_{i} *$ for any $\mathbf{a}, \mathbf{b} \in l_{2}(\mathfrak{A})$. If $\mathfrak{A}$ has an ideńtity then $l_{2}(\mathfrak{U})$ is countably generated. The Hilbert $\mathfrak{A}$-module is standard for all comitably generated Hilbert $\mathfrak{A}$-moduli in the sense of G. G. KaSParov's Stabilisation theorem $\left[9:\right.$ Th. 2].. Let us describe the inner structure of $l_{2}(\mathfrak{Q t})$.' Denote by $\left\{\mathbf{e}_{i}\right\}_{i \in \mathbb{N}}$ the canonical orthonormal basis of $l_{2}(\mathfrak{Q})$.

Lem ma 4.1 : Let $\mathfrak{A}$ be a $C^{*}$-algebra with identily and $l_{2}(\mathfrak{A})$ be the standurd connlably generated Hilbert $\mathfrak{A}$-module. 'Then the map $\psi: f \in l_{2}(\mathfrak{A})^{\prime} \rightarrow\left\{f\left(\mathbf{e}_{i}\right)^{*}\right\}_{i \in \mathbb{N}}$ is a bijecion between $\cdot l_{2}(\mathfrak{A})^{\prime}$ and the set of all sequences $\mathbf{a}=\left\{a_{i}\right\}_{i \in \mathbb{N}} \subset \mathfrak{A}:$ with the property $\sup _{N} \| a_{1} a_{1}^{*}+\cdots$

$+a_{N} a_{N}^{*} \|<+\infty .:$ Moreover, $\psi$ maps all bounded module maps of the form $\varphi(\mathbf{a})$ $=\langle\cdot, \mathbf{a}\rangle, \mathbf{a} \in l_{2}(\mathfrak{X})$, into the characterizing element a and vice versa.

$$
\text { Corollary 4.2: We have }\|f\|^{2}=\lim _{N}\left\|\sum_{i=1}^{N} f\left(\mathbf{e}_{i}\right)^{*} f\left(\mathbf{e}_{i}\right)\right\|_{\mathbb{2}}^{v} \text { for any } f \in l_{2}(\mathfrak{A})^{\prime} \text {. }
$$

The statements of Lemma 4.1 and Corollary 4.2 are mentioned by [ 16$]$ and by [5] without proof. Since the proof is easy it will be omitted. The following theorem is an extension of [6: Prop. 3, Prop. 4] to the non-commutative case. It was first proved by, the author [7: Th. 22] with global $C^{*}$-algebraical methods and, independently, by $\mathrm{O}$. G. Fúrprov [5] considering maximal commutative $C^{*}$-subalgebras. We reproduce here the proof from [7] in an ameliorated variant.

Theorem 4.3: Let $\mathfrak{A}$ be a $C^{*}$-algebra. The following conditions are equivalent:

(i) $\mathfrak{A}$ is finile-dimensional.

(ii) $l_{2}(\mathfrak{U})$ is self-dual.

(iii) For each $\mathbf{a} \in l_{2}(\mathfrak{A})$ the series $\sum\left\|a_{\mathrm{i}}\right\|^{\mathfrak{2} 2}$ converges.

Proof: We start with a simple observation. If the $C^{*}$-algebra $\mathfrak{A}^{\ddot{H}}$ finite-dimensional, it contains an identity. If $l_{2}(\mathfrak{A})$ is self-dual, then the bounded module map $h$ defined. by the formula $h(\mathfrak{a})=a_{1}$ for any $\mathfrak{a}=\left\{a_{\mathfrak{i}}\right\}_{i \in \mathbb{N}} \in l_{2}(\mathfrak{U})$ belongs to $l_{2}(\mathfrak{A}) \subseteq l_{2}(\mathfrak{A})^{\prime}$. Thus, $\mathfrak{A}$ must contain an identity.

A'ssume now that $\mathfrak{X}$ contains an'identity. The Hilbert $\mathfrak{A}$-module $l_{2}(\mathfrak{A})$, is self-dual if and only if any norm-bounded increasing directed sequence of self-adjoint positive elements of $\mathfrak{A}$ is fundamental relative to the norm-topology of $\mathfrak{A}$, cf. Lemma 4.1 . Equivalent to this condition is that all linear positive functionals on $\mathfrak{A}$ are normal, i.e., that the universal representation of $\mathfrak{A}$ is normal and, equivalently, that $\mathfrak{A}$ is 
reflexire as a Banach space. The latter is true if and only if $\mathfrak{A}$ is finite-dimensional. The equivalence of the conditions (i) and (iii) follows from a proposition of A. DvoRETZKY and C. A. Rogers [4], which can be found in [17: Prop. 3.4.1, Prop. 1.6.2]

As a corollary we can extend [19: Th. 3] to these criteria:

Proposition 4.4: Let $\mathfrak{A}$ be a $C^{*}$-algebra. The following conditions are equivalent:

(i) ' $\mathfrak{A}$ is finite-dimensionàl.

(ii) Any Hilbert $\mathfrak{A}$-module $\mathbf{M}$ is self-dual.

Moreover, if the $C^{*}$-algebra $\mathfrak{A}$ is commutative and unital (or, respectively, is a $W^{*}$-algebra), there exists a third equivalent condition:

(iii) Any Hilbert $\mathfrak{Q}$-module $\mathbf{M}$ is' $\mathfrak{A}$-reflexive.

Proof: The first item follows from [19: Th. 2] and from Theorem 4.3 above. To prove the second one we consider a compact space $\dot{K}$ consisting of infinitely many - points. We denote by $C(K)$ the set of all continuous complex-valued functions on $K$ and, respectively, by $C_{0}(K)$ the set of all $f \epsilon^{\circ} C(K)$ satisfying $f(x)=0$ at a, certain fixed accumulation point $x \in K$. The sets $\dot{C}(K)$ and $C_{0}(K)$ are both $C^{*}$-algebras, where the latter is a two-sided ideal in $C(K)$. We define on $C(K)$ the usual inner product $\langle\cdot, \cdot\rangle_{\mathfrak{O}}$. Then $\mathbf{M}=\left\{C_{0}(K),\langle\cdot, \cdot\rangle_{\mathfrak{I}}\right\}$ turns into a Hilbert $C(K)$-module for which the connection $\mathbf{l}^{\prime}=\mathbf{M}^{\prime \prime}=C(K)$ holds. Hence, $C_{0}(K)$ is not $\mathfrak{A}$-reflexive.

If $\mathfrak{A}$ is an infinite-dimensional $W^{*}$-algebra, the counter-example is given by Theorem 3.2 and Theorem 4.3

Proposition 4.5: Let $\mathfrak{A}$ be a $W^{*}$-algebra or, respectively, a commutative unital $C^{*_{-}}$ algebra." The following two conditions are equivalent:

(i) $\mathfrak{U}$ is finite-dimensional.

(ii) For any. Hilbert $\mathfrak{A}$-module $\mathbf{N}$ and any Hilbert $\mathfrak{A}$-submodule $\mathbf{M} \subseteq \mathbf{N}$ there holds $\mathbf{N} .=\mathbf{M} \oplus \mathbf{M}^{\perp}$.

Proof: If $\mathfrak{A}$ is an infinite dimensional $W^{*}$-algebra, both $l_{2}(\mathfrak{A})$ and $l_{2}(\mathfrak{A})^{\prime}$ are noncoinciding Hilbert $\mathfrak{A}$-moduli, where $l_{2}(\mathfrak{A}) \subset l_{2}(\mathfrak{A})^{\prime}$ and $l_{2}(\mathfrak{A})^{\perp}=\{\mathbf{0}\}$. -If $\mathfrak{A}=C(K)$ is a commutative unital $C^{*}$-algebra; the Hilbert $\mathfrak{A}$-module $\left\{C_{0}(K),\langle\cdot, \cdot\rangle_{\mathfrak{P}}\right\}$ described in the, proof of Proposition 4.4 can be viewed as a Hilbert, $\mathfrak{A}$-submodule of $\mathfrak{A}$ : But, $C_{0}(K)^{\perp}=\{0\}$ in this case and they do not coincide. Referring to [19: Th. 1, Lemma 3] we finish the proof

Cörollary 4.6: If $\mathfrak{A}$ is a $C^{*}$-algebra with an infinite-dimensional two-sided $W^{*}$. ideal, the standard countably generated $\dot{H}$ ilbert $\mathfrak{Q}\left(-\right.$ module $l_{2}(\mathfrak{U})$ is neither self-dual nor A-reflexive.

This follows from Theorem 3.2 and Theorem 4.3. As a further application we describe below the structure of self-dual and $C^{*}$-reflexive countably generated Hilbert $W^{*}$-moduli. That any finitely generated Hilbert $C^{*}$-module is self-dual was recalled in Corollary 2.9. Similarly, any Hilbert $C^{*}$-module over a finite-dimensional $C^{*}$-algebra is self-dual, cf. Corollary, 2.9 .

Proposition $4.7:$ Let $\mathfrak{A}$ be an $W^{*}$-algebra and $\mathbf{M}$ be a $C^{*}$-reflexive (and, hence, selfdual) countably generated Hilbert $\mathfrak{A}-$ module. There are two possibilities for the structure of $\mathbf{M}$ and of $\mathfrak{A}$ :

(i) $\mathbf{N}$ is finitely generated and $\mathfrak{A}$ is urbitrary.

(ii) $\mathbf{M}$ is the direct sum of a finilely generated Hilbert $\mathfrak{A}$-module and of a counlably. generated Hilbert $\dot{\mathfrak{B}}$-module, where $\mathfrak{B}$ is a finite-dimensional two-sided $C^{*}$-ideal in $\mathfrak{\mathfrak { A }}$.

If the $W^{*}$-algebra $\mathfrak{A}$ has no finite-dimensional two-sided $C^{*}$-ideuls, any countably infinitely generated Hilbert $\mathfrak{A}$-module is non-self:dual and non-C*-reflexive. 
Proof: Let $\left\{\dot{\mathbf{x}}_{i}\right\}_{i \in \mathbb{N}}$ be the system of generators of $\mathbf{M}$ as $\mathfrak{A}$-module. By [14: Prop. 3.11] and $\left[\right.$ [18: Lemma 6.7] there exists another system ${ }^{\prime}$ of $^{\prime}$ generators $\left\{\mathbf{y}_{i}\right\}_{i \in \mathbb{N}}$ of $\mathbf{M}$ deduced from the first one such that $\left\langle y_{i} ; y_{i}\right\rangle=p_{i}=p_{i}{ }^{2} \neq 0$ and $\left\langle y_{i}, y_{j}\right\rangle=0$ for any $i \neq j$. Denote by $L_{\mathfrak{A}}\left(\grave{y}_{i}\right)$ the norm-closed $\mathfrak{A}$-linear hull of $\mathbf{y}_{i}$. The Hilbert $\mathfrak{A}$-module $\left\{L_{\mathfrak{A}}\left(\mathbf{y}_{i}\right),\langle\cdot,:\rangle\right\}$ is self-dual. Therefore, it is isomorphic to $\left\{\mathfrak{A} p_{i},\langle\cdot, \cdot\rangle_{\mathfrak{A}}\right\}$ by Theorem 3.8 and $\left\langle L_{\mathfrak{I}}\left(\mathbf{y}_{i}\right), L_{\mathfrak{U}}\left(\mathbf{y}_{i}\right)\right\rangle$ is a two-sided $W^{*}$-ideal in $\mathfrak{A}$ for any $i \in \mathbb{N}$. Consequently, we get that $\mathbb{M}$ is isomorphic to the Hilbert $\mathfrak{A}$-module -

$$
\mathbf{N}=\left\{\mathbf{x}=\left\{x_{i}\right\}_{i \in \mathbb{N}}: x_{i} \in \mathfrak{U} p_{i}, \sum x_{i} x_{i}^{*} \text { is }\|\cdot\|_{\mathfrak{A} \text {-coverging }}\right\} \text {. }
$$

Now we try to reach a situation in which the product of any two projections $p_{i}, p_{j}$. $(i<j)$ of our choice is a projection $r$ if and only if $r=p_{j} \neq 0$, For this end we use an inductive process of construction. First, fix the projection $p_{1}$ and check all products $r_{k}=p_{i} p_{k}(k \in \mathbb{N})$. If $r_{k}$ is a projection for a certain $k \in \mathbb{N}$ and if $r_{k} \neq 0$, then replace $p_{1}$ by the sum $\left(p_{1}+p_{k}-r_{k}\right)$ and $p_{k}$ by $r_{k}$. If $r_{k}=0$, then replace $p_{1}$ by $\left(p_{1}+p_{k}\right)$ and exclude $p_{k}$ from our choice: Finishing this first step we deal with the pairwise products $r_{k}{ }^{\prime} \doteq p_{2} p_{k}^{\prime}(k \in, \mathbb{N})$ with the first factor $p_{2}$ in the same way. This process is continued by induction. We remark that the claimed inductive process on the projections $\left\{p_{k}: k \in \mathbb{N}\right\}$ of $\mathfrak{A}$ is compatible with the module operations inside $\mathbf{N}$ and $\mathbf{M}$, respectively:

Suppose now there exist more than finitely many two-sided $W^{*}$-ideals $\left\langle\mathfrak{A} p_{i}, \mathfrak{A} p_{i}\right\rangle$ of our reconstructed choice being infinite-dimensional. Then $\mathbf{M}^{\prime} \neq \mathbf{M}$ by Theorem 3.8 and 'Theorem 4.3. Suppose' there exists no finite-dimensional two-sided $C^{*}$-ideal $\mathfrak{B}$ in $\mathfrak{U}$ containing all finite-dimensional $W^{*}$-ideals $\left\langle\mathfrak{A} p_{i} ; \mathfrak{A} p_{i}\right\rangle$ of our reconstructed choice. Then $\mathbf{M}^{\prime}+\mathbf{M}$ by Theorem 3.8 and Theorem 4.3

$\therefore$ Finally, we state the main problem arising if these results are to be extended to the case when $\mathfrak{A}$ is not necessarily a $W^{*}$-algebra. W. L. Paschke [14: Th. 3.2] nóted without proof referring to [20] that the $\mathfrak{A}$-valued inner product of a Hilbert $\mathfrak{A}$-module lifts on to the dual Banach $\mathfrak{A}$-module even if $\mathfrak{A}$ is a commutative $A W^{*}$-algebra. The question is: What are the conditions needed that this oan be done for any Hilbert $\mathfrak{A}$-module over a certain $C^{*}$-algebra $\mathfrak{A}$ ? One condition is that $\mathfrak{A}$ must be an $A W^{*}$ algebra [16: Prop. 1.1]. It.seems to be necessary that $\mathfrak{A}$ must be monotonically complete and possess an analogy of the $w^{*}$-topology coinciding with the topology of order convergence on bounded directed nets of self-adjoint elements of $\mathfrak{A}$, cf. Theorem.4.3. Solving this problem one could get general criteria of self-duality and $C^{*}$-reflexivity of Hilbert $C^{*}$-moduli.

\section{REFERENCES}

[1] Bratteli, O., and D. W, RoBrsson: Operator algebras and quantum statistical mechanies, part 1 (Texts and Monographs in Physics). New York-Heidelberg-Berlin: SpringerVerlag 1979.

[2] DúprE, M. J., and P. A. Frlmore: Triviality theorems for Hilbert modules. In: Topics in modern operator theory (Timişoara/Herculane 1980; edś. C. Apostol, R. G. Douglas, B. Sz. - Nagy and D: Voiculescu) (Operator theory.: Adv. Appl.,2). Basel-Boston, Mass.: Birkhïuser Verlag 1981, p. 71-79.

[3] DuPRE, M. J., and R. M. Gillette: Banach burdles, Banach modules and automorphisms" of $C^{*}$-algebras (Research Notes in Mathematics 92). Boston-London-Melbourne: Pitman Adv. Publ. Program/1083.

[4] Dvoretzky, A.; and C. A. Rogers: Absolute and unconditional convergence in nórmed ' linear spaces. Proc. Nat. Acad. Sci. USA 36 (1950), 192-197. 
[:] Филиппов, О. Г.: ' O $C^{*}$-алгебрах $A$, над которыми гильбертов модуль $l_{2}(A)$ автолуален. Вестиик Моск. Гос. Ун-та. Серия I : Математика. Механика 4 (1987), 74- 76.

[6] Frank, M.: A set of maps from $K$ to $\operatorname{End}_{A}\left(l_{2}(A)\right)$ isomorphic to $\operatorname{End}_{A(K)}\left(\left(l_{2} A(K)\right)\right)$. Applications. Annuls Global Anal. Geom. 3 (1985); $155-171$.

[7] FrANK, M.: Self-duality and $C^{*}$-reflexivity of Hilbert $C^{*}$-moduli. KMU-CLG-proprint Leipzig: Karl-Marx-Universität 1986.

[8] Goldstine, H: H., and L. P. Honwitz: Hilbert space with non-associative scalars, IJ. Math. Ann. 164 (1966), $291-316$.

[9] Kasparov, G. G.: Hilbert $C^{*}$-modules: Theorems of Stinesspring and Voiculescu. J. Oper. Theory 4 (1980), $133-150$.

[10] Loyses, R. M.: Linear operators in VH-spaces. Trans. Amer: Math. Soc. 166 (1965), 167 to 180 .

[11] Мйщенко, А. С., и А. Т. Фоменио: Индекс эллиптических операторов нал $C^{*}$ алгебрами. Изњ. Акащ. Наук СССР, Серин мат., 43 (1979), 831-859.

[12] Мищғнко, А. С.: Банаховы алгебры, псевдодифференциальные опер́торы и। ,приложения к $K$-теории. Успехи мат. наук $34(1979) 6,67-79$.

[13] Мищенко, А. С.: Представления компактных групи в гиліббертовых модулях нал $C^{*}$-алгебрами. Тр. мат. ин-та Акад. Наук СССР 166 (1984), 161-176.

[14] Paschik, W. L.: Inner product modules over $B^{*}$-algebras. Trans. Amer. Math. Soc. 18ะ; (1973), $443-468$.

[15] Paschke, W. I.: The double $B$-dual of an inner product module over a $C^{*}$-algébra $B$. Can. J. Math. $26(1974), 1272-1280$.

[16] Pascirke, W. L.: Inner product modules arising from compact automorphism groups of - von Neumann algebras. Trans. Amer. Math. Soc. 294 (1976), 87-102.

[17] Pretsch, A.: Nukleare lokalkonvexe Räume. Berlin: Akademie-Verlag 1969.

[18] Riffred, M. A.: Morita equivalence for $C^{*}$-algebras and $W^{*}$-algebras. J. Pure Appl. Alg. $5(1974), 51-96$.

[19] Saworotyow, P. P.: A generalized Hilbert space. Duke Math. J. 35 (1968), '191-197.

[20] Widom, H.: Embedding in algebras of type I. Duke Math.'J. $\mathbf{9 3}$ (1956), 309-324.

Minusthripteingang:.22, 03. 1988; in revidierter Fissung 01.03.1989

VERFASSER:

Dr. Michafl Frank

Sektion Mathematik der Karl-Marx-Universität

Karl-Marx-Platz

DDR - 7027 Leipzig 